4

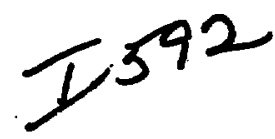
Particle Trajectories in Equilibrium Magnetic Fields

D. V. Anderson

R. H. Cohen

J. R. Ferguson

B. M. Johnston

C. B. Sharp

P. A. Willmann

June 30, 1981 


\title{
ORBXYZ: A 3D Single-Particle Orbit Code for Following Charged- Particle Trajectories in Equilibrium Magnetic Fields
}

\author{
D. V. Anderson \\ R. H. Cohen \\ J. R. Ferguson \\ B. M. Johnston \\ C. B. Sharp \\ P. A. Willmann
}

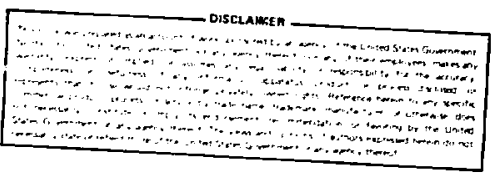

Manuscript date: June 30, 1981

\section{LAWRENCE LIVERMORE LABORATORY Universisy of California - Livermore, California $\bullet 94550$}




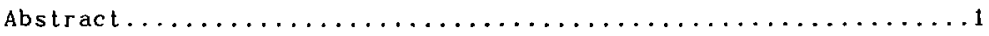

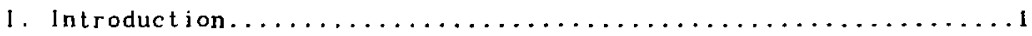

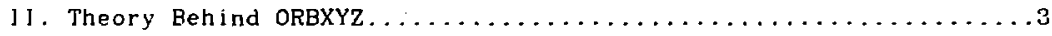

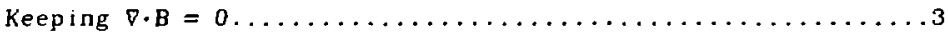

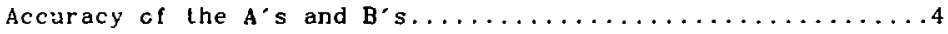

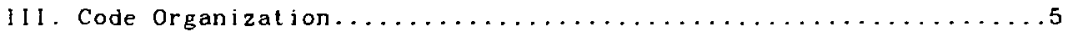

Generating Spline Coefficients.................5

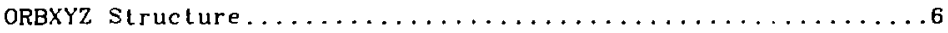

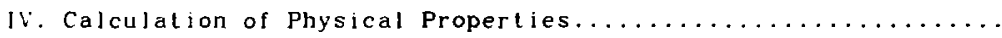

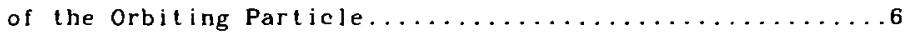

V. Results for a High-Beta MFTF Equilibrium Field............1

vi. Conclusions................................

Elimination of linwanted $\nabla \cdot B \neq 0$ Effects.............

Desıgn of Jon Beam Probes.....................

Refprences...........................

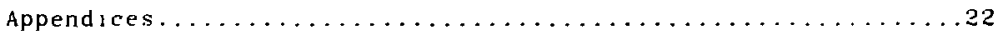


ORBXYZ: A 3D Single Particle Orbit Code for Following

Charged Particle Trajectories in Equilibrium Magnetic Fields

\section{Abstract}

The single particle orbit code, TIBRo, has been modified extensively to improve the interpolation methods used and to allow use of vector potential fjelds in the simulation of charged particle orbits on a 3D domain. A 3D cubic B-spline algorithm is used to generate spline coefficients used in the interpolation. Smooth and accurate $f$ ield representations are obtained. When vector potential fields are used, the 3D cubic spline interpolation formula analytically generates the magnetic field used to push the particles--this field has $\nabla \cdot B=0$ to computer roundoff. When magnetic induction is used the interpolation allows $\nabla \cdot B \neq 0$, which can lead to significant nonphysical results. Presently the code assumes quadrupole symmetry, but this is not an essential feature of the code and could be easily removed for other applications. Many detajls pertaining to this code are given on microfiche accompanying this report.

\section{Introduction}

This report is the fourth and last in a series of UCRL 'eports which describe the numerical models used to perform studies of cnarged particle confinement in finite beta plasma equilibrium fields. The previous reports describe the codes ABCXYZ, ${ }^{1}$ TPSIC, ${ }^{2}$ and VEPEC; ${ }^{3}$ this report describes the code ORBXYZ. Briefly they perform the following tasks:

1. ABCXYZ: On a 3D Cartesian grid we generate the vector potential and magnetic induction field components due to an arbilrary current carrying coil arrangement. In particular we frequently use the yin-yang set found in minimum-B mirror devices such as 2XIIB or the MFTF-B plugs.

2. TPSIC: A version of this routine, SETORB, generates the trilinear cubic spline coefficients for the fjelds generated by ABCXYZ. Two modes of operation are available:
a. We spline the vector potential--the $A^{\prime} \mathbf{s}$.
b. We spline the magnetic induction--the $\mathrm{B}^{\prime} \mathrm{s}$. 
3. VEPEC: For the same 3D grid we take the spline representation of the vacuum vector potentials calculated in ABCXYZ and SETORB and solve the plasma equilibrium problem with this vacuum field. Vector potentials due to the plasma currents are calculated and added to the vacuum field values to produce the total equilibrium field vector potential. Magnetic induction is carried as a subsidary, derived field array. A finite element (FEM) approach which represents the vector potentials as an array of trjcubic spline coefficients is used to give high-order accuracy.

4. ORBYYZ: Single charged particles are followed in a 3D domain according to the Lorentz force. At each orbit point $B$ is evaluated from $B=\nabla \times A$ (or $B=B$ ) depending on whether we $\operatorname{splined~} A^{\prime} s$ (or $B^{*} s$ ) Detalled information regarding the magnetic moment, phase angles. etc. is generated and plots of the partjcle trajectory are generated.

ORBXYZ is a modification of the TlBRO code 4 and differs from it in three important respects as shown in Table I below:.

Table 1. Features of the TIBRO and ORBXYZ Codes.

\section{T I BRO}

Grid

Interpolation

Fields Used

MAFCO Option
Cylindrical

"Ind order" One-sided Lagrange Interpolation

$B^{\prime} \mathrm{s}$

Yes
ORBXYZ

Cartesian

Tricubic Spline

$B^{\prime} s$ or $A^{\prime} s$

No

In TIBRo the equations of motion. Eq. (1), were always formulated in rectangular coordinates but the interpolation grid was cylindrical. Problems associated with singularities at the axis are avojded in VEPEC by using Cartesian coordinates. It js convenient and accurate to use the same grid in ORBXYZ as in VEPEC, so rectangular coordinates are used throughout ORBXYZ except in some output djagnostics. The MAFCO option $r \in f \in[s$ to the generation of fjeld components due to cojls as evaluated \% various complete and incomplete eljjptic integral routines. It is $765 \%$ time consuming for realistic coil configurations but was necessary 1. I!ERO as it is very accurate compared to the use of gridded fields. Is CEEXYZ the tricubic spline interpolation is sufficiently accurate tilyt 25 dded representations of the field (either $A^{\prime} s$ or $B^{\prime} s$ ) are 
sufficient for studying vacuum $f$ ields. Other differences also exist, mainly in lne area of output diagnostics (here OREXYZ has more options)

This report first discusses the rationale for building ORBXYZ in Section II. Section III sketches the code organization while in IV varjous diagnostics useful in confinement studies are discussed. In $V$ results are obtained showing how this approach avoids the $\nabla \cdot B \neq 0$ problem. Conclusions are in VI. The appendices A, B, C, and D are reserved for a source listing, typical input, printed output, and plots respectively. Much of the material of the afpendices has been put on the $f$ jche to be found in the pocket on the inside back cover of this report.

\section{Theory Behind ORBXYZ}

This code. Iike its predecessor TIBRO, integrates the equations of motion by using thehigh-order ordinary differential equation solver $\operatorname{GEAR}^{5}, 6$ to evolve:

$$
\begin{aligned}
& \frac{d x}{d t}=v, \\
& \frac{d v}{d t}=a, \\
& a=\frac{e}{m c}(v \times B) .
\end{aligned}
$$

The particle is pushed, so io speak, from point to point and the timestep used in the ordinary differential equation solver is automalically adjusted to satisfy an error criterion. By making this error criterion small enough and assuning $B$ is known exactly then the trajectory can be made arbitrarily close to the exact trajectory--except for computer roundoff error.

Keeping $\nabla \cdot \mathrm{B}=0$

A difficulty with TIBRO is that sometimes B is known only on a grid and interpolation is required. One must live with the errors of this interpolation. Not only is the one-sided "second order" Lagrange interpolation inaccurate, but it also produces fields seen by the particle in which $\nabla \cdot B \neq 0$.

A major application of single particle orbjt codes is the study of the confinement of particles in realistic mirror equilibrium fields. 
For mirrors, confinement is due to the approximate invariance of the magnetic moment $\mu$. From the theory of adiabatic mirror confinement ${ }^{7}$ the instantaneous evolution of $\mu$ is given by:

$$
\frac{1 \mathrm{~d} \mu}{\mu \mathrm{dt}}=-\frac{\mathrm{v}_{-1}}{\mathrm{~B}} \nabla \cdot \mathrm{B}-\frac{\left(\mathrm{v}_{\perp}^{2}+2 \mathrm{v}_{\| 1}^{2}\right)}{\mathrm{v}_{\perp}} \frac{|\mathrm{B} \times \nabla \mathrm{B}|}{\mathrm{B}^{2}} \cos \psi
$$

plus other terms. ${ }^{7}$ Here $\psi$ is the instantaneous phase angle between $v_{-}$ and the $B \times \nabla B$ direction. Physically, and in reasonable analytical representations of $B$, the first term vanishes since $\nabla \cdot B=0$. Numerically, truncation errors of interpolation can introduce $\nabla \cdot B \neq 0$ which can result in significant nonphysical contributions to d $\mu / d t$. The remedy is to use interpolation methods that have $\nabla \cdot B=0$ as a constraint. Imposing this constraint on typical interpolation formulae leads to conditions on the integrals of $B$ which suggest using an integrated quantity for performing the interpolation. The vector potential is the obvious candjdate because if we have a smooth enough formula for A then

$$
\nabla \cdot \mathrm{B}=\nabla \cdot(\nabla \times \mathrm{A})=0
$$

from the usual Identity of vector analysis. Clearly A must have two cont ınuous derivatives in each independent variable $x, y$, and $z$ in order to satisfy Eq. (3) everywhere. The interpolation formula which clearly satisfles this is a tricubic spline. On earh grid cell a tricubic polynomial is used to "analytically" represent the vector potential. On neifhboring cells the representation has continuity up to and including the 2nd derivatives across the cell boundaries. A rather general method for constructing tricubic spline interpolation formulae has been presented in the TPSIC report. 3 To preserve Eq. (3) the magnet lc field used in the code at the orbit point is obtained by anajytically calculating the curl from the $A$ 's. This is done easily since the representation is polynomial.

Accuracy of the $A^{\prime} s$ and $B^{\prime} \cdot s$

As is well known one loses at least an order of accuracy by taking a derivative of a given representation. That is, if the $A$ 's have errors of order $h^{4}$ in the tricubic spline formula, then the $B^{\prime} s$ have errors of order $h^{3}$ or less. At first glance an orbit code using B's directly from the grid might seem to be more accurate (of course the $\nabla \cdot B \neq 0$ problem will occur). To resolve this question we must refer to the equilibrium codes themselves. Nearly every equilibrium code solves for some kind of 
potential rather than for B directly. This relates to the difficulty of inverting the curl found in Ampere's law

$$
\nabla \times B=\frac{4 \pi}{c}-J .
$$

In fact the curl operator does not have an inverse. At the convergence of an equiljbrium code the $B^{\prime}$ s are obtained by using the appropriate finite difference (FID) or finite element (FEM) operator on the representation of the potential. At this stage the order of the error is reduced. In VEPEC we solve

$$
\nabla^{2} \mathbf{A}=\frac{4 \pi}{c} \mathbf{J}
$$

and we ke.s the $A^{\prime} s$ in their spline representation to use in ORBXYZ. $B^{\prime}$ 's calculated in VEPEC are also avajlable to ORBXYZ for making comparisons. To be more specific we list the order of error found for the $A^{\prime} s$ and $B^{\prime} s$. If the $A^{\prime} s$ are represented by cubic splines then

$$
\text { Quanti:y }
$$

$$
\begin{array}{ll}
A(t r i c u b i c s p l i n e s) & O\left(h^{4}\right) \\
B=\nabla \times A \text { (analytical from splines) } & O\left(h^{3}\right) \\
B=" \nabla \times " A \text { (centered finite difference) } & O\left(h^{2}\right)
\end{array}
$$

\section{Error}

Since VEPEC and ORBXYZ currently use the first two of these (FEM combination), orbiting particles see errors in $B$ of order $O\left(h^{3}\right)$. Many other codes, including the finite difference (FID) version of VEPEC, ${ }^{8}$ have errors in the $B^{\prime} s$ of order $O\left(h^{2}\right)$ since they usually finite difference the "potential" function with a centered difference formula.

\section{III: Code Organization}

\section{Generating Spline Coefficients}

OREXYZ expects input in the form of arrays of spline coefficients for the $A^{\prime} s$ or the $B^{\prime} s$. SETORB, which is a modification of the TPSIC code, is used to invert the linear system of equations defining the spline representation, thus obtaining the B-spline coefficients. Three 
inversions, one for each vector component, are performed. Input data for SETORB is shown in Appendix B. It specifies the types of symmetry and boundary conditions to be used. In the case where vacuum fields of coils are to be used, SETORB is run to transform the arrays of $A$ 's or $B^{\prime} s$ on a grid to the corresponding errays of spline coefficients. The vector potential equilibrium code, VEPEC, directly gentrates the spline coefficient arrays for the $A^{\prime} s$ but computes field arrays for the $B^{\prime} s$. To follow particles in equilibrium fjelds with the B option then requires a pass through SETORB to convert the $B^{\prime} s$ to the ir spline representation.

\section{ORBXYZ Struc lure}

In the MAIN subroutine NAMELIST data is read vhich specifies the number of trajectorjes to be run along with other parameters including the initial condjtions for the orbit. Each call to TRAJECT calculates a single trajectory from time zero up to the specified time limit for that particle. On each subsequent trajectory a new data "card" is read in giving the new initial conditions. TRAJECT performs three basjc tasks:

1. It calculates the $\mathrm{f}$ ield at the orbit point by calling routines which use the spline package already described in the TPSIC ${ }^{2}$ report.

2. A multitude of diagnostics are calculated. In particular the instantaneous guiding center position is calculated a:1d stored for plotting. Criteria for the adiabaticity theory are extracted as well. Conditions for calling output routines and for stopping the trajectory are tested and acted upon. Arrays to be used by an optional movie postprocessor are written to disk.

3. DRIVE, the GEAR package 5.6 ordinary differential equation solver, Is called to advance the particle to the next orbit pount.

In crude outline this is the structure of the code. Appendix A gives the source listing from which further details can be gleaned.

\section{V. Calculation of Physical Properties of the Orbiting Particle}

So $f$ ar we have shown how ORBXYZ integrates the single particle equation of motion in the magretjc field or vector potential representation. Also we have given a crude outline of how the code is organized. Now we show in detail how the output diagnostics are calculated. The primary trajectory we calculate is, of course, the particle's path. of secondary interest is the guiding center trajectory. The instantaneous guiding center locus is calculated as 


$$
x^{g c}=x+\frac{m c}{q} \frac{(v \times B)}{B^{2}}
$$

On the primary trajectory a fundamental quantity $\mu$, the magnetic moment, is calculated directly,

$$
\mu=\frac{m(v \times B)^{2}}{2}=\frac{w_{1}}{B} .
$$

To calculate the gyro-averaged magnetic moment $\langle\mu\rangle$ we sum up the "N" within one gyloperjod and djvide by the number of timesteps taken in the gyroperiod. It is appropriately prorated over the first and last time steps as the gyroperiod is usually not an exact integer multiple of the timestep. Let the orbit points in a gyroperiod be numbered 1 through $x$ and let $t^{(n)}$ represent the time at each orbit point. Also define

$$
\delta t^{(n)}=t^{(n+1)}-t^{(n)} .
$$

Let $t^{s}$ and $t^{\prime}$ denote the beginning and ending times for the gyroperiod. Require

$$
\begin{aligned}
& t(0)<t_{E}<t(1) . \\
& t(N)<t_{I}<t(N+I) .
\end{aligned}
$$

Since the instantaneous gyrofrequency charges even within a gropertod wo must defone the gyroperiod carefully. In the cont inuum

$$
3=\frac{1}{2 \pi} \int_{t_{s}}^{t_{1}} \omega(t) d t
$$

Is used lo determine $t_{f}$. Since $t_{s}$ is assumed known from having ralculated the previous gyroperiod we use

$$
\begin{aligned}
& T_{\text {gyro }}=t_{f}-t_{s}, \\
& T_{\text {inst }}(t)=2 \pi
\end{aligned}
$$


In the finjte representation Eq. (10) is replaced by

$$
1=\frac{\omega^{(1)}+\omega_{s}}{4 \pi} \delta t_{s}+\frac{\omega^{(N)}+\omega_{f}}{4 \pi} \delta t_{f}+\sum_{n=1}^{N-1} \frac{\omega^{(n)}+\omega^{(n+1)}}{4 \pi} \delta t(n),
$$

where

$$
\begin{aligned}
& \delta t_{s}=t^{(1)}-t_{s}, \\
& \delta t_{f}=t_{f}-t^{(N)}, \\
& \omega_{s}=\omega^{(0)}+\frac{\omega^{(1)}-\omega^{(0)}}{\delta t^{(0)}}\left(t_{s}-t^{(0)}\right), \\
& \omega_{f}=\omega^{(N)}+\frac{\omega^{(N+1)}-\omega^{(N)}}{\delta t^{(N)}}\left(t_{f}-t^{(N)}\right) .
\end{aligned}
$$

Only $t_{f}$ is unknown. Solving Eq. (13) for $t_{f}$ we use Eq. (11) to get $\tau_{\text {gy }}$ o

Having defined the gyroperiod we can calculate an averaged $\mu$ (or other gyro-averaged quantities). Continuously.

$$
\langle\mu\rangle=\frac{\int_{t_{s}}^{t_{f}} \frac{\mu d t}{\tau(t)}}{\int_{t_{s}}^{t_{f}} \frac{d t}{\tau(t)}}
$$

and discretely.$$
\frac{\mathrm{t}^{(1)}-\mathrm{t}_{s}}{\delta \mathrm{t}^{(0)}}(0)+\frac{\mathrm{t}_{\mathrm{f}}-\mathrm{t}^{(\mathrm{N})}}{\delta \mathrm{t}^{(N)}} \mu(\mathrm{N})+\sum^{N-1} \mu^{(\mathrm{n})}
$$$$
\langle\mu\rangle=\frac{n=1}{N+\frac{\imath^{(1)}-t_{s}}{\delta t^{(0)}}+\frac{t_{f}-t^{(N)}}{\delta t^{(N)}}} .
$$

To initialize a new gyroperiod after obtaining the point $t^{(N+1)}$ we 
replace

$$
\frac{t^{(1)}-t_{s}}{\delta t^{(0)}}=1-\frac{t_{f}-t^{(N)}}{\delta t^{(N)}}
$$

We also calculate the canonjcal momentum on the primary trujectory and particularly the canonical angular momentum $P_{\theta}$

$$
\begin{aligned}
& P=m v+\frac{q}{c} A \\
& P_{\theta}=x P_{y}-y P_{x} .
\end{aligned}
$$

Several other quantities are beeded at the particle position. The phase of particle is an important quantity in the formuia for du/dt which we ne rite out in full. ${ }^{7}$ We will use $\mu:^{*}$ to distinguish it from the $\mu$ defined in Eq. (7).

$$
\begin{aligned}
\frac{1 \mathrm{~d} u^{*}}{\mu^{*} \mathrm{dt}} & =-\frac{v^{\prime \prime}}{\mathrm{b}} \nabla \cdot \mathrm{B}-\frac{v_{\perp}^{2}+2 v_{\mathrm{u}}^{2}}{v_{\perp}} \rho_{\perp} \cos (\psi)+\frac{2 v_{\mathrm{n}}^{2}}{v_{\perp}} \rho_{\mathrm{J}} \cos (\bar{\psi}) \\
& -v_{11}\left[\mathrm{e}_{1} \cdot\left(\mathrm{e}_{1} \cdot \nabla\right) \mathrm{b}-\mathrm{e}_{2} \cdot\left(\mathrm{e}_{2} \cdot \nabla\right) \mathrm{b}\right] \cos (2 \psi) \\
& +v_{i n}\left[\mathrm{e}_{1} \cdot\left(\mathrm{e}_{2} \cdot \nabla\right) \mathrm{b}+\mathrm{e}_{2} \cdot\left(\mathrm{e}_{1} \cdot \nabla\right) \mathrm{b}\right] \sin (2 \psi)
\end{aligned}
$$

is "andlytically" evaluated at each orbit point. Here the following definitions ard jdentificalions are used: 


$$
\begin{aligned}
& \mathrm{b}=\frac{\mathrm{B}}{\mathrm{B}} ; \text { unit vector along } \mathrm{B} \\
& \mathrm{v}_{\perp}=-(\mathrm{b} \times(\mathrm{b} \times \mathrm{v})) ; v_{\perp}=\left|\mathrm{v}_{\perp}\right|=\left(\frac{2 \mu \mathrm{B}}{\mathrm{m}}\right)^{1 / 2} \\
& \mathrm{v}_{\mathrm{H}}=\mathrm{b} \cdot \mathrm{v} \\
& \rho_{\perp}=|\mathrm{B} \times \nabla \mathrm{B}| \mathrm{B}^{-2} ; \text { inverse radial scale length of } \mathrm{B} \\
& \rho_{J}=|\mathrm{B} \times(\nabla \times \mathrm{B})| \mathrm{B}^{-2} ; \text { inverse radial scale length of } \mathrm{J} \\
& \cos (\psi)=\frac{(\mathrm{B} \times \mathrm{v}) \cdot(\mathrm{B} \times \mathrm{VB})}{|(\mathrm{B} \times \mathrm{v})||(\mathrm{B} \times \nabla \mathrm{B})|}
\end{aligned}
$$

; alpha-gyropháse$$
\sin (\psi)=\frac{(B \times(B \times v)) \cdot(B \times \mathrm{BB})}{|\mathrm{B} \times(\mathrm{B} \times \mathrm{v})||(\mathrm{B} \times \mathrm{BB})|}
$$$$
\cos (\bar{v})=-\frac{(\mathrm{B} \times(\mathrm{B} \times \mathrm{v})) \cdot(\mathrm{B} \times(\nabla \times \mathrm{B}))}{|\mathrm{B} \times(\mathrm{B} \times \mathrm{v})||\mathrm{B} \times(\nabla \times \mathrm{B})|} ; \text { beta-gyrophase }
$$$$
e_{2}=\frac{b \times \nabla B}{|b \times \nabla B|} \text {; unit vector in drif: direction }
$$$$
e_{1}=e_{2} \times b \text { : unit normal lo drift surface. }
$$

The code calculates both $\mu$ and " which differ only because of the finite timeslep used in the GEAR integration. In the $t$ ime continuum $\mu^{*}=\mu^{*}$ the code has checked this b: making the timestep arbitrar $1 \mathrm{y}$ small. All of the quantities in Eq. (17) are evaluated on the primary trajectory. Most of the quantitics on the right side of these expressions are known at the particle position. but the gradients of $B$ must be calculated accurately within the spline approximation. All of the other factors involving these gradients are trivial io evaluate once the nine elements of the dyad $\nabla B$ are known. The function subprogram FUNT is capable of producing not only the interpolated function but the analytical evaluation of the partial derivatives of the spline formula as well. For example the dyad element $\partial_{y} B_{x}$ is calculated as

$$
\partial_{y} B_{x}=\partial_{y} B_{x}^{\text {spline }}
$$

or

$$
\partial_{y} B_{x}=\partial_{y}\left(\partial_{y} A_{z}^{\text {spline }}-\partial_{z} A_{y}^{s p l i n e}\right)
$$


in the $B$ and $A$ options respectively. Here $B_{x}^{s p l i n e}$ and $A_{z}^{s p l i n e}$ and A $y$ pline are the scalar $t$ ields represented in the tricubic spline furmula. The routine FUNT analytically applies the operators $\partial_{y}$ or $\partial_{y} \partial_{y}$ and $\partial_{y} \partial_{z}$. etc. to the spline formula to generate the required elements of the dyad $\nabla B$. Further discussion of these features in the tricubic spline package is presented in the TPSIC 2 report.

When Eq. (17) is integrated over a bounce period. the change for jump) in $\mu$ during that interval can be put in the form

$$
\Delta \mu=\left(\frac{\mu v}{v_{\perp}}\right) e^{-x / \varepsilon} \cos (\psi)
$$

where: is the phase of the partucle when the instantaneous gu:ding center is at a minimum of B along its path. Various examples of analytical expressions for $\Delta \mu$ of this type are given by Cohen et $a l^{7}$. We can cajculate $\hat{n} u$ independently in the code to sheck the validity of Eq. (20). For this purpose we use

$$
i_{u}=\left.\langle\mu\rangle\right|_{z_{+}}-\left.\langle\mu\rangle\right|_{z_{-}}
$$

where $z_{+}$and $z_{-}$refer to the bounce positions.

These bounce positions are obtained from the quantity $v \cdot B$ along the guiding center trajectory and define $z_{+}$or $z_{-}$as the positions of the guiding center just before $v \cdot B$ changes sign. $\left.\langle u\rangle\right|_{z}$ is defined as the ( $\mu$ already evalunted in the gyroperiod which included the bounce or turning point.

Other diagnostics may be added in similar fashion.

\section{Results for a High-Beta MFTF Equilibrium Field}

Already the ORBXYZ code has been used routinely in several applicat icis. For example, it was used to follow representative particles born in a mirror device from a neutral beam to learn how certain neutral beam aiming schemes would influence the axial and radial extent of the plasma generated. In another study it was used to show the very striking phenomenon of orbital resonances responsible for anomalous particle losses in the RECE-Berta experiment.9.10 And it has been used to confirm predictions of the analytic nonadiabaticity theory for misror machines. ${ }^{7}$ Allso, tests were performed several years ago showing the clear superiority of ORBXYZ over the TlBRo code when both were run with the gridded option. 8 in $\mathrm{fact}$ it was found that the gridded ORBXYZ was sufficiently accurate to obviate the MAFCO option. 
Somewhat suprisingly it turned out that ORBXYZ ran faster than TIBRO for the gridded versions in spjte of the fact that the tricubic interpolation used in ORBXYZ required 64 terms to the 27 terms needed in the "second order" forward Lagrange interpolator used in TIBRO. It turns out the Lagrange interpoiation gave a significant field ripple which caused the Gear integrator to choose a smaller time step thus slowing the TIBRO code.

The results we present here are obtained for a high-beta MFTF- $B$ plug equilibrium as compuled by the vector potential equilibrium code, VEPEC. We distinguish between the current version of VEPEC $^{3}$ which employs tricubic B-spline finite elements (FEM) and an older verston which used finite differences (FID). For all of the cases shown we used the same equilibrium compuled from a pressure profile due to Rensink 10 with a central beta of .63. Particles were typically launched at the midplane within a few degrees of the loss cone boundary. For high energy particles we studied 4.4 Mev alpha particles (somewhat more energetic than a typical $D-T$ fusion reaction product). In Figs. 1-3 we hare plotted a projection of an orbit segment, the instantaneous magnetic moment history durung this orbit segment. and the gyro-averaged magnetic moment from a longer orbit respectively for these alpha particles. 


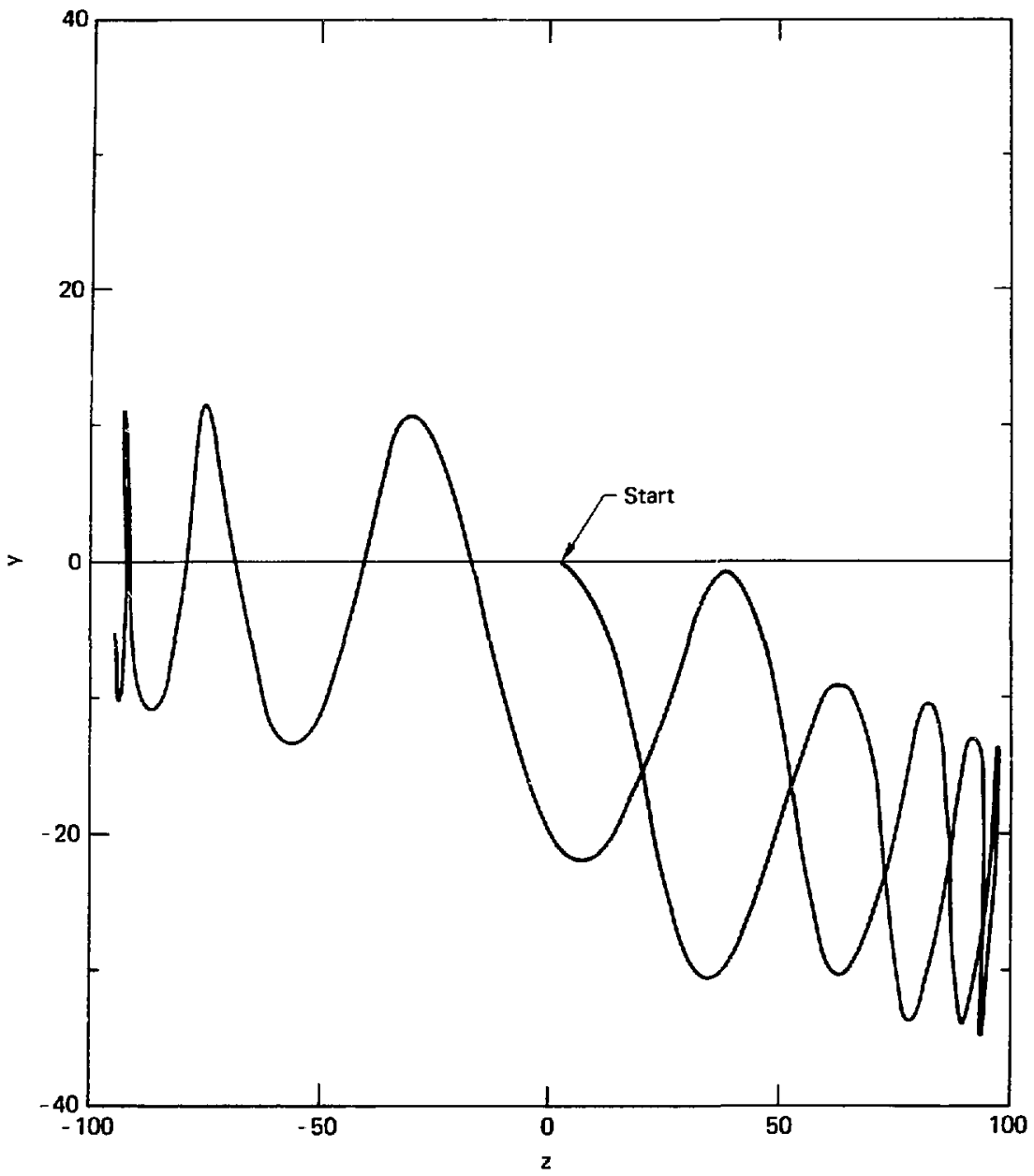

Figure 1. The $y-z$ plane projection of the trajectory of a 4.4 Mev alpha particle is shown for a high-beta MFTF-B plug equilibrium configuration. 


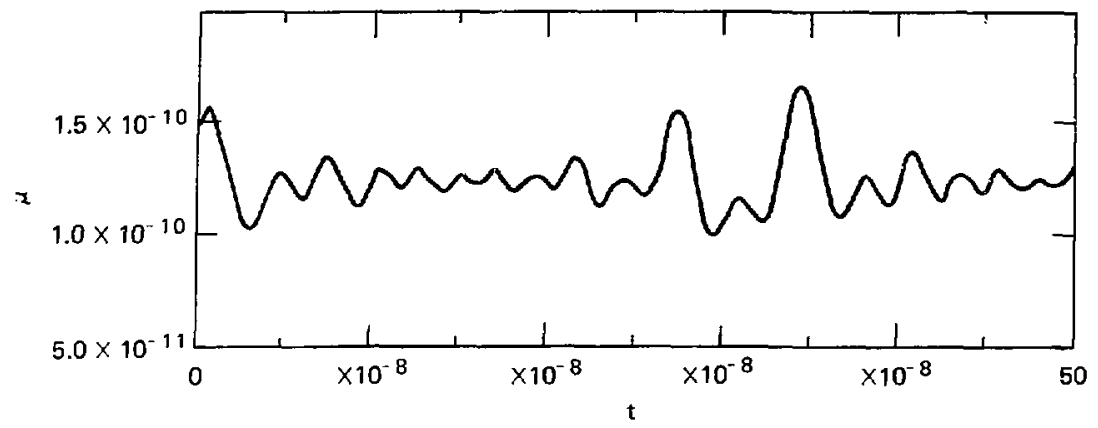

Figure 2. Instantaneous magnetic moment of the $4.4 \mathrm{Mer}$ alpha partzcle is displayed as a function of time.

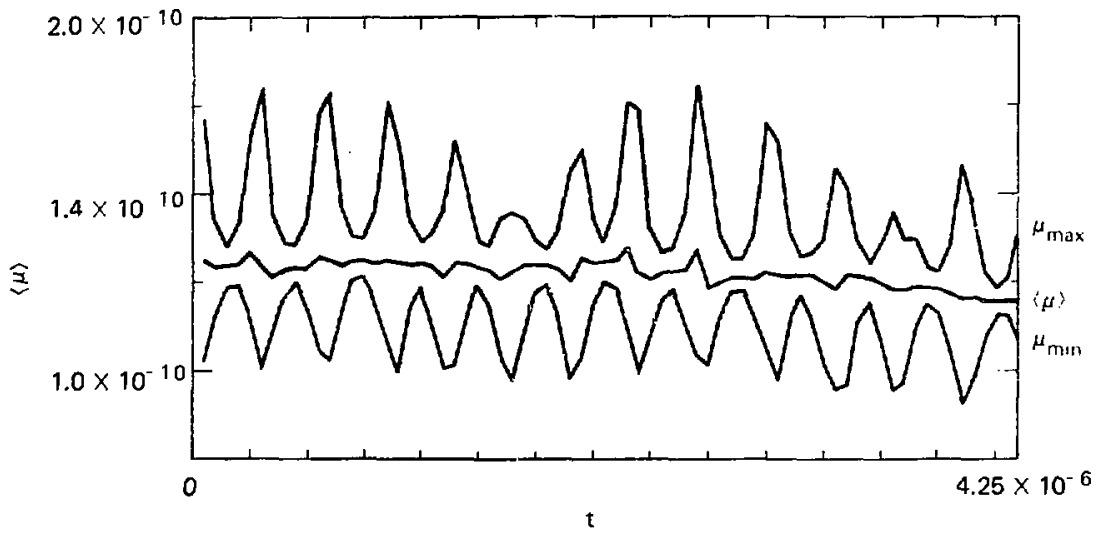

Figure 3. Gyro-averaged $\mu$ logether with its envelope functions ari shoun for tize 4.4 Mev alpha particle. We note $\mu$ is not we $i$ conscricd leadiug to a short confinement time for this particle. 


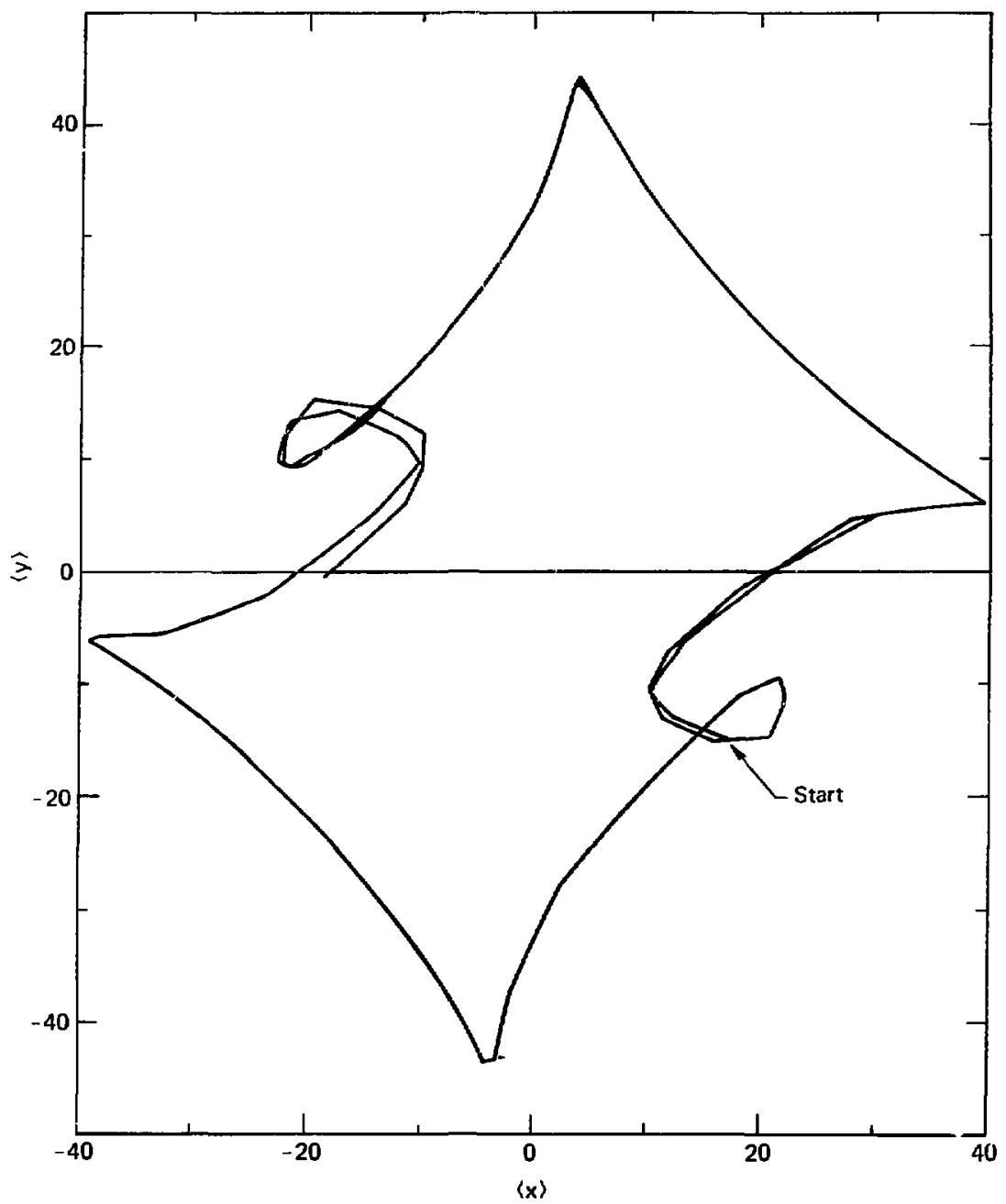

Figure 4. The $x-y$ plane projection of the guiding center locus of the 4.4 Mev alpha shows a complicated yet regular "drift" surface.

15 
In Fig. 4 wo plot the $x-y$ plane projection of the guiding center trajeclory. This diagnostic allows one to study properties such as that of omnigenity. For these high energies the $\nabla \cdot B$ term is very small even when the $B^{\prime} s$ are used.

Both the fields chosen for interpolation ( $A^{\prime} s$ or $B^{\prime} s$ ) and the numerical method used in the equilibrium calculation may affect the orbits. We have studied four different conbinations, a! f for $50 \mathrm{kev}$ deuterons, as given in Table II below. Both the FID version of VEPEC ${ }^{8}$ and the FEM version ${ }^{3}$ are compared. We present code diagnostics showing how $\mu$ varies during several bounces in the simulation. A random part of $\Delta \mu$ is computed and used to estimate a confinement $t$ ime-the $t$ jme for the particle to random walk over to the loss cone. We have also plotted the time history of the gyro-averaged magnetic moment and its envelope functions in Fig. 5. Note how the FID method combined with the B's gives pathological behavior. In Table II we note this case had a severely degraded confinement time. When B's were used but obtained from the FEM equjJjbrium or whenever $A^{\prime} s$ were used (FID or FEM). good consistent risults were obtained. Differences among the successful methods (spatial truncation errors and physical stochastic effects) resulted in some difference in the $\Delta \mu / \mu$ values found. This variation is to be expected if the $\Delta \mu^{\prime} s$ are the result of a random process. 
Table 11 . Comparisoti of confinement diagnostics of the four methods for a 50 kev deuteron in $\alpha \beta_{1}=0.63 \mathrm{MFTF}-B \mathrm{plug}$. We assume $\Delta \mu / \mu=.4$ determines the loss cone boundary. These estimates crudely presumed that $\Delta \mu / \mu$ was the result of a purely random process.

Number of

Transits $n$

FID B's FIDA's FEM B's FEM A's

$\begin{array}{lllll}10 & 11 & 8 & 8\end{array}$

$\Delta \mu / \mu$ afler

n Transits

1. $2 \times 10^{-2}$

$4.9 \times 10^{-4}$

$6.0 \times 10^{-4}$

$1.1 \times 10^{-3}$

Ef fective Random $\Delta \mu / \mu$

for one Transit

$3.8 \times 10^{-3}$

$1.5 \times 10^{-4}$

$2.1 \times 10^{-4}$

$3.9 \times 10^{-4}$

Coufinement in

Number of Transits

$1.7 \times 10^{4} \quad 7.1 \times 10^{6}$

$3.6 \times 10^{6}$

1. $1 \times 10^{6}$

Corresponding

Conf inement $\mathrm{T}$ ime (sec)

0.019

12.2

7.52

2.30

Fractional $\nabla \cdot B$

Error to d $\mu / d t$

$10^{-1}$

$10^{-14}$

$10^{-3}$

$10^{-14}$ 

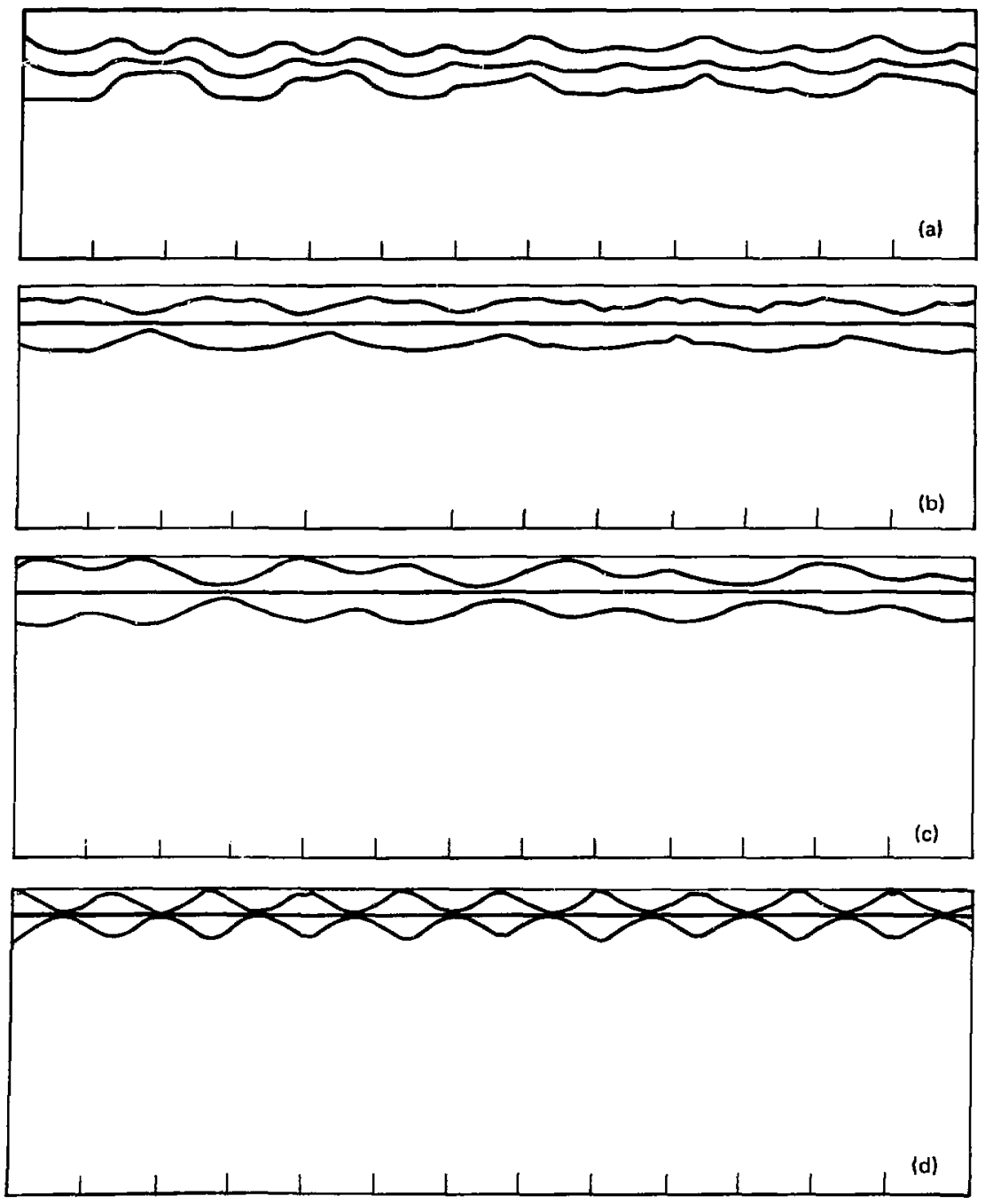

$$
\mu_{\max }{ }^{(t)},(\mu(t)), \mu_{\min }{ }^{(t)}
$$

Figure 5. In $a, b, c$, and $d$ we give the history of the gyro-averaged $\mu$ and its envelope functions for a 50 kev deuteron using the $F I D-B, F I D-A$. FEM-B, and the FEM-A methods respectively. The FID-B results are visually poor. 


\section{Conclus lons}

\section{Elimination of Unwanted $\nabla \cdot B \neq 0$ Effects}

The poor result obtained for the FID $B^{\prime} s$ is seen in Table II to correspond to a large value (error) of $\nabla \cdot B$. This type of error cannot. happen with the A's regardless of their origin (FID or FEM) and for these two cases Table II shows roundof $f$ level $\nabla \cdot B$ values. The FEM $B^{\prime} s$ do not insure $\nabla \cdot B=0$ but the $h i$ iner accuracy of this method keeps it small enough to not noticeably af fect the result. We can understand this by a streightforward analysis of the errors in each code; these are summarized in Table III.

Table III. Comparison of finite difference and finite element errors.

$\begin{array}{lll}\text { Order of Errors } & \text { FlD } & \text { FEM } \\ \text { Equilibrium Calculation of A's } & h^{3} & h^{4} \\ \text { B's Generated Therefrom } & h^{2} & h^{3} \\ \text { ORBXYZ A's } & h^{3} & h^{4} \\ \text { ORBXYZ B's from "A" Option } & h^{2} & h^{3} \\ \text { ORBXYZ B's from "B" Option } & h^{2} & h^{3} \\ \text { ORBXYZ } \nabla \cdot B \text { from " } A \text { " Option } & 0 & 0 \\ \text { ORBXYZ } \nabla \cdot B \text { from "B" Option } & h^{1} & h^{2}\end{array}$

The errors are expressed as a power of the grid spacing $h$. The Jowest order (biggest) error ( $h^{1}$ ) occurs for the quantity $\nabla \cdot B$ from the FID equilibrium and ORBXYZ using the "B" option. The FEM B's at the grid points are precisely consistent with a solenoidal divergenceless field $B$, but in the FID VEPEC the $B^{\prime} s$ are already inconsistent with the solenoidal property. When FEM B's are given to the ORBXYZ code and resplined, small higher order $\left(\mathrm{h}^{2}\right)$ errors are found in $\nabla \cdot B$.

A minimum conclusion is that vector potentjals must be generated in the equilibrium calculation; otherwise there is no way to construct $B^{\prime} s$ such that $\nabla \cdot B=0$ or is very small. If one insists on splining $B$ 's in ORBXYZ, then they must be obtained from a finite element representation of the $A^{\prime} s$ in the equilibrium code. The simplest and most accurate method to follow is to use the FEM methods to obtain equlibrium $A^{\circ} s$ and then spline A's for the ORBXYZ calculation. 


\section{Design of I on Beam Probes}

Cutler modif jed ORBXYZ to a version called ORB4 which has been used by Hormady ${ }^{2}$ and others for the design of ion beam probes on magnetie fusion confinement experiments. It was used successfully both for tokomak ${ }^{13}$ and tandem mirror ${ }^{14}$ applications.

\section{References}

1. D. V. Anderson. J. Breazeal, C. H. Finan, and B. M. Johnston. The ABCXYZ Code for Computing Vector Potential and Magnetic Fields from General Current Elements, Lawrence Livermore Laboraloiy Report UCRL-52029 (Sept. 1976).

2. D. V. Anderson. The Three-Dimensional Tensot Product Spline Interpolation Code (TPSIC), Lawrence Livermore Laboratory UCRL-51958 (Jail. 1976).

3. D. Y. Anderson, J. Breazeal, and C. B. Sharp, VEPEC: A BD Vector Potential Equilibrium Code for High-Beta, Minimum-B Plasma Confinement, Lawrence Livermore National laboratory Report UCRL-53099 (Jan. 1981).

4. J. H. Foote, R. P. Freis, and D. F'uss, Controlled Thermonuclear Research Annual Report, Lawrence Livermore Laboratory Report. UCRL-50002-67 (1967).

5. C. W. Gear, Numerzal Initial Value Problems in Ordinary Differential Equations iPrentice Ha]l, Englewood Cliffs. N. J., 1971).

6. A. C. Hindmarsh, CEAR, ar Ordinary Differential Equation Solver. Lawrence Livermore Laboratory Report UCID-30001, Rev. 3 (1974).

i. R. H. Cohen, G. Rowlands, and J. H. Foote, Phys, Fluids, 21, 627 (i978).

8. D. V. Anderson, B. M. Johnston, and J. Breazea], Numerical Studies of Particle Confinement in High-Beta Equilibrium Mirror Plasmas. Lawrence Livermore Laboratory Report UCRL-7761 Abstract (Dec. 1975); Proc. 2nd European Conf. on Computational Physics, Max-Planck Institut. Garching. Federal Republic of Germany (April 197.5).

9. R. H, Cohen, D. V. Anderson, and C. Sharp, Orbital Resonances in Quadrupole Stabilized Mirror Configurations, Lawrence Livermore Laboratory Report UCRL-80960 Abstract (March 1978); Proc. Annual 1978 Controljed Fusion Theory Conference, Gatlinburg, Tennessee (April 1978). 
10. R. H. Cohen, D. V. Anderson, and C. Sharp. Phys. Rev. Letters, 19 (Nov. 6. 1978).

11. M. E. Rensink, Bull, APS, 21, 1187 (1976).

12. Timothy A. Cutler and R. S. Hornady, An lon Trajectory Code, 2nd Topical Conference on High Temperature Plasma Diagnostjcs, A. P. S., Bull. A. P. S. 23m (May-June 1978).

13. R. S. Hornady, private communication. Hickok and co-workers used ORB4 to design an ion beam probe for the tokomak at Rensselaer Polytechnic Institute.

14. G. Hollock, K. Saadatmond, J. Lewis, W. Jennings, R. Hickok, and R. Hornady, A Heavy Ion Beam Probe for the Central Cell of TMX. Bul1. A. P. S, 23. (Sept. 1978). 


\title{
Appendices
}

\section{Appendix A}

To allow the reader to obtain very detailed information about the ORBXYZ code we have provided its FORTRAN source in this appendix. We have avoided a bulky document by providing it on Fiche 1. For completeness the source of SETORB, used to create the spline arrays. is also given on $\mathrm{F}$ jche 1 .

\section{Appendix $B$}

The short data input il iles to ORBXYZ and SETORB are given on Fiche 2. Each of these codes operates off larger input djsk files as well. On these the Iield arrays are stored either as spline coefficien. wrays or as the $f$ ield component arrays. The structure of these large input disk files can be determined from the source ijsting or from the VEPEC , eport. ${ }^{3}$

\author{
Append $\mathrm{i} \times \mathrm{C}$ \\ Printed output from ORBXYZ is given on Fiche 3.
}

\section{Appendix D}

Plots are displayed on Fiche 4. We note that all of the input and output shown in these appendices came from one run: the source shown was the one compiled to produce these results. This run was for a $4.4 \mathrm{Mev}$ alpha particle launched at a radius of $20 \mathrm{~cm}$ in the midplane. 